\title{
Marsupialização como tratamento definitivo de cistos odontogênicos: relato de dois casos
}

\author{
Marsupialization as definitive treatment of odontogenic cysts: \\ report of two cases
}

Gustavo Nascimento de Souza Pinto* Jéssica Araújo Figueira* Eduardo Sanches Gonçales ${ }^{* * *}$

Eduardo Sant'ana ${ }^{* * * *}$

Elen de Souza Tolentino ${ }^{* * * * *}$

\section{Resumo}

Objetivo: a marsupialização é uma opção de tratamento dos cistos, no qual uma janela cirúrgica comunicando com a cavidade bucal, suturada junto à mucosa adjacente, é aberta para o esvaziamento progressivo do conteúdo interno da lesão, acarretando em sua descompressão e consequente diminuição. Por definição, a técnica é acompanhada por um segundo tempo cirúrgico para exérese da lesão remanescente. Este artigo objetiva apresentar uma revisão de literatura a respeito da técnica de marsupialização e relatar dois casos de cistos odontogênicos de diferentes origens, nos quais a marsupialização foi realizada como tratamento definitivo, com resultados satisfatórios em ambos os casos. Relato de caso: a) homem, 22 anos, apresentou uma área radiolúcida, bem definida, envolvendo a coroa do dente 48, sugestiva de cisto dentígero. O paciente foi submetido à extração do dente 48 e à marsupialização, com total remissão da lesão; b) mulher, 38 anos, encaminhada pelo endodontista devido à presença de uma lesão radiolúcida, bem definida, com halo radiopaco no periápice do dente 21 , sugestivo de cisto de origem inflamatória. O tratamento escolhido foi a marsupialização, resultando em reparo ósseo e remissão da lesão. Considerações finais: a marsupialização como tratamento definitivo para lesões císticas é uma abordagem que leva à menor morbidade ao paciente e também à preservação de estruturas anatômicas nobres, porém o tema é pouco abordado na literatura. É importante salientar que esse procedimento isolado não apresenta previsibilidade e a evolução de cada caso deve ser avaliada.

Palavras-chave: Cisto dentígero. Cisto periapical. Cirurgia bucal.

\section{Introdução}

A marsupialização é considerada uma forma de tratamento muito comum para alguns tipos de cistos e tumores odontogênicos. Não é raro que alguns autores confundam os termos marsupialização e descompressão, utilizando-os de maneira errônea. Ambas as técnicas têm por objetivo reduzir a pressão interna da lesão com a retirada de fluído, que leva, consequentemente, à redução progressiva da lesão, para posterior enucleação. O que difere as duas técnicas é que na descompressão é necessária a instalação de um artifício (dispositivo) para manutenção da abertura cirúrgica ${ }^{1}$. A técnica cirúrgica é considerada simples: realiza-se uma incisão geralmente circular e cria-se uma janela ampla, para comunicação da lesão com a cavidade oral, que é su-

\footnotetext{
Mestrando em Clínica Integrada, Universidade Estadual de Maringá, Maringá, PR, Brasil.

Graduanda de Odontologia, Universidade Estadual de Maringá, Maringá, PR, Brasil.

Professor doutor, Departamento de Estomatologia, área de Cirurgia e Traumatologia Bucomaxilofacial, Faculdade de Odontologia de Bauru, Universidade de São Paulo, Bauru, SP, Brasil.

* Professor doutor, Departamento de Estomatologia, área de Cirurgia e Traumatologia Bucomaxilofacial, Faculdade de Odontologia de Bauru, Universidade de São Paulo, Bauru, SP, Brasil.

**** Professora doutora, Departamento de Odontologia, Universidade Estadual de Maringá, Maringá, PR, Brasil.
} 
turada junto à mucosa adjacente, e ao retirar parte da cápsula da lesão, ela já fornece material para o exame histopatológico ${ }^{2}$.

Essa técnica é muito utilizada em casos de lesões extensas, visando à preservação de estruturas nobres próximas ao campo operatório, como, os dentes, feixes vásculo-nervosos e espaços aéreos. Geralmente é necessário um segundo tempo cirúrgico para total remoção da lesão, após a diminuição de seu tamanho. $\mathrm{O}$ número de trabalhos abordando a marsupialização como tratamento definitivo é escasso, porém existem relatos da eficácia dessa técnica quando ela foi eleita como tratamento definitivo $^{1-3}$.

Diante da alta prevalência dos cistos nos maxilares, técnicas mais conservadoras para os pacientes devem ser eleitas, visando ao melhor prognóstico bem como à preservação de estruturas anatômicas importantes. A marsupialização vem sendo apontada como um procedimento cirúrgico mais conservador, uma vez que permite a descompressão cística acarretando em regressão da lesão. Alguns estudos têm mostrado que essa técnica, que, por definição, deve ser acompanhada de enucleação, pode ser realizada como tratamento definitivo, acarretando em menor morbidade ao paciente, já que um segundo tempo cirúrgico não é necessário ${ }^{1,4}$. Nesse contexto, o objetivo deste trabalho é caracterizar a marsu- pialização como tratamento definitivo para lesões císticas bem como relatar dois casos clínicos de cistos odontogênicos de diferentes origens nos quais a marsupialização como tratamento único obteve sucesso.

\section{Relato de caso}

Ambos os pacientes foram atendidos na Clínica de Estomatologia da Faculdade de Odontologia de Bauru, da Universidade de São Paulo, e assinaram termos de consentimento livre e esclarecido permitindo a coleta dos dados.

\section{Caso 1}

Homem, leucoderma, 22 anos, apresentou-se com queixa de odontalgia na região mandibular direita. Clinicamente a região em si não revelava nenhuma alteração, o paciente não apresentava nenhuma assimetria facial e não relatou nenhuma alteração sistêmica. A radiografia panorâmica evidenciou uma área radiolúcida, bem definida, de aproximadamente $30 \mathrm{~mm}$ envolvendo a coroa do dente 48. A tomografia computadorizada de feixe cônico (TCFC) revelou fenestração da tábua óssea lingual (Figuras 1: A e B).

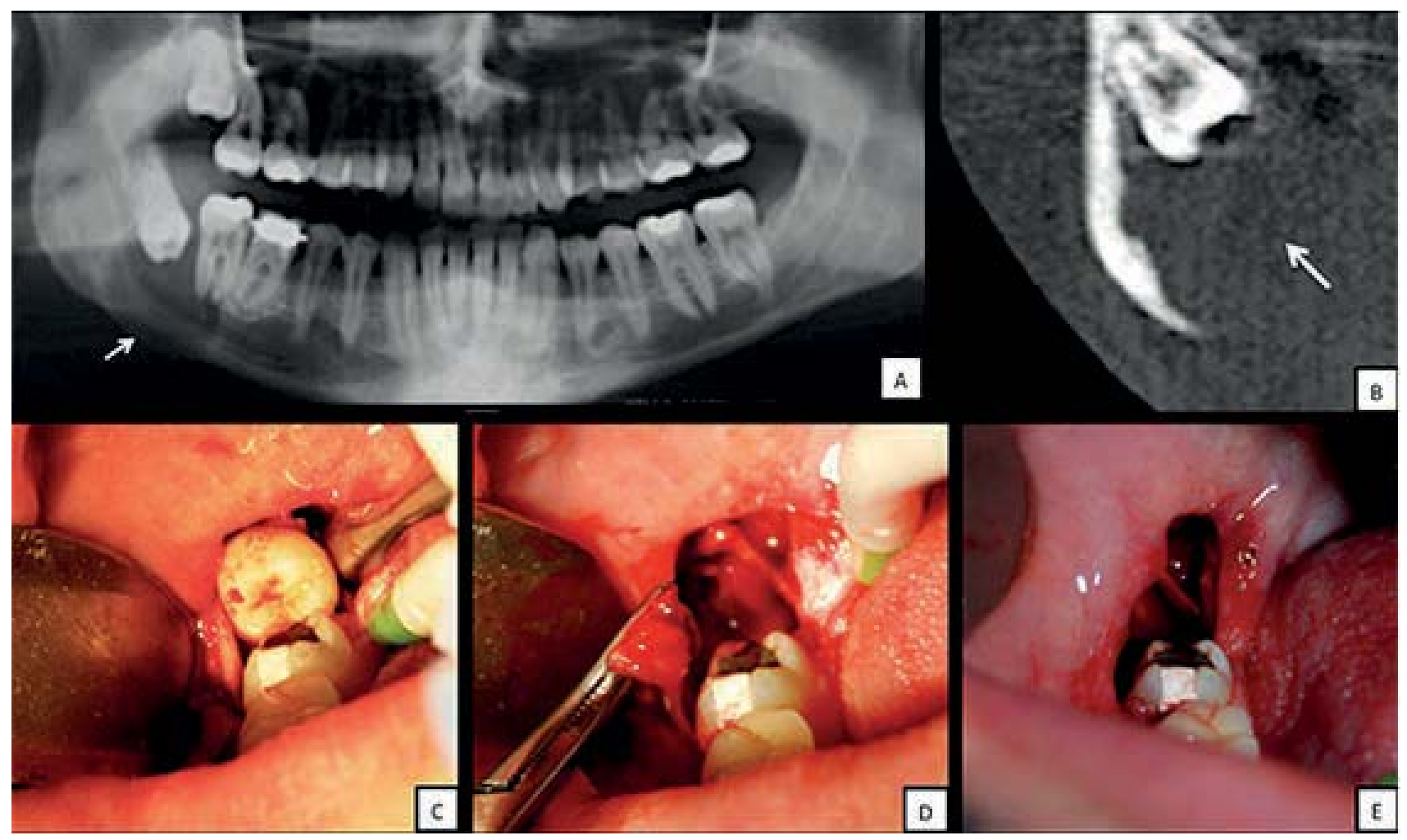

Figura 1 - Radiografia panorâmica inicial evidenciando área radiolúcida envolvendo a coroa do 48 ( $A$, seta); TCFC (reformatação coronal) evidenciando perda óssea da tábua óssea lingual (B, seta); Exodontia do dente 48 (C); Cápsula da lesão (D) e loja cirúrgica mantida aberta $(E)$

Fonte: todas as figuras são de elaboração dos autores. 
Diante das características clínicas e radiográficas apresentadas, a hipótese diagnóstica foi de cisto dentígero. A odontalgia era decorrente de uma restauração profunda no dente 47.

$\mathrm{O}$ paciente foi então submetido a um procedimento cirúrgico, visando à exodontia do dente $48 \mathrm{e}$ à enucleação da lesão. Porém, durante a cirurgia, não houve colaboração do paciente e, diante das dificuldades de manejo, optou-se pela manutenção da janela cirúrgica suturada às mucosas adjacentes, caracterizando a técnica de marsupialização (Figura $1: \mathrm{C}, \mathrm{D}, \mathrm{E})$.
Durante a primeira semana, o paciente compareceu às consultas diárias para irrigação da área com clorexidina $0,12 \%$ e soro fisiológico. Depois, ele foi orientado a realizar a higiene rigorosa do local com soro fisiológico ${ }^{1}$.

O fragmento da lesão foi enviado para análise microscópica, que revelou achados que confirmaram a hipótese de cisto dentígero.

Aos oito meses de pós-operatório, o paciente foi submetido novamente a uma TCFC, e foi observada uma considerável neoformação óssea da tábua lingual. No controle de dois anos, a análise radiográfica evidenciou um total reparo ósseo no local da lesão (Figura 2).

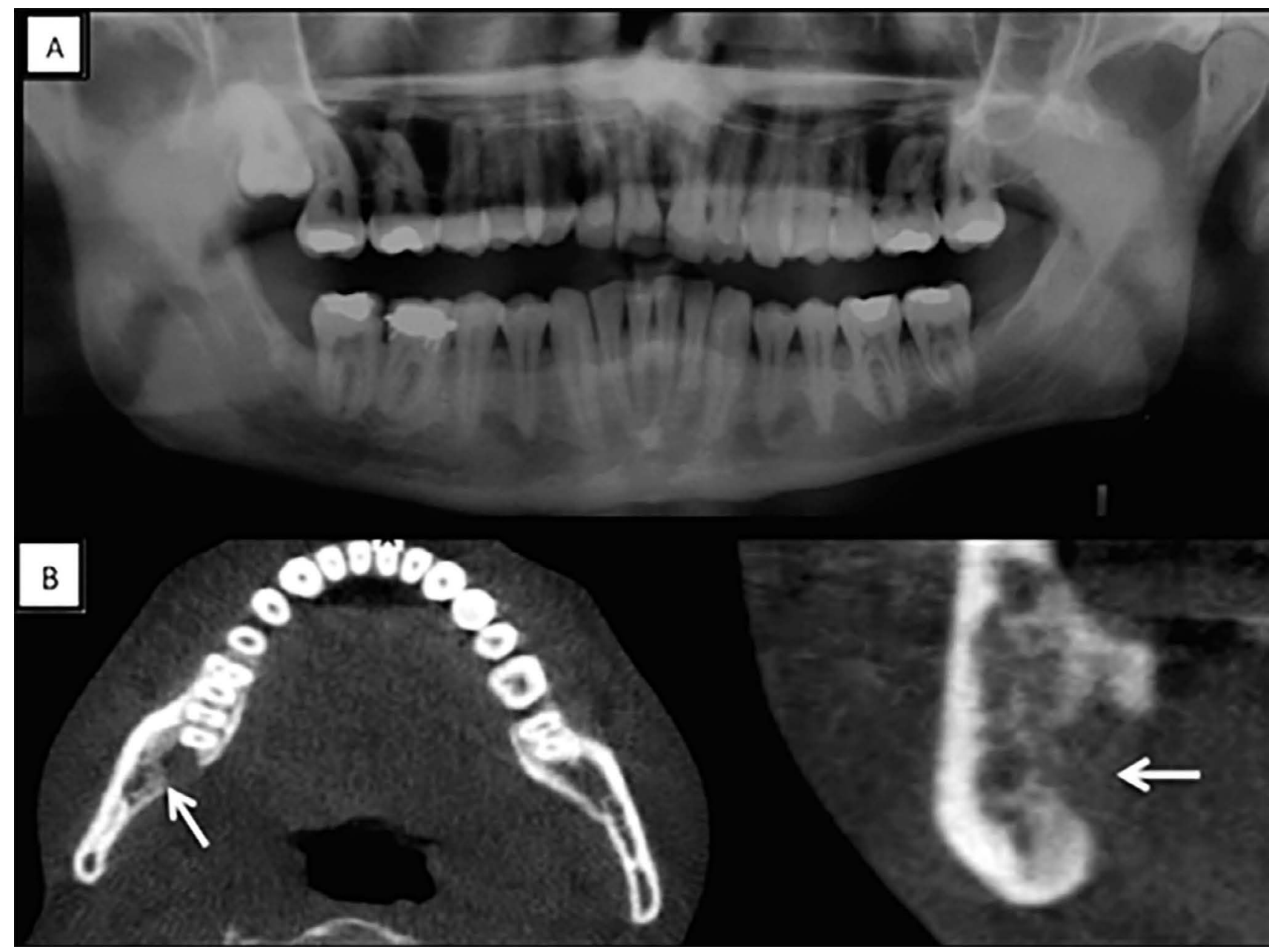

Figura 2 - Radiografia panorâmica no pós-operatório de dois anos mostrando completo reparo ósseo na região (A); TCFC no pós-operatório de oito meses e reformatações axial e coronal demonstrando significativa neoformação óssea na cortical lingual (B, setas)

\section{Caso 2}

Mulher, leucoderma, 38 anos, encaminhada pelo endodontista que constatou a presença de uma lesão radiolúcida de aproximadamente $40 \mathrm{~mm}$, bem definida e com halo radiopaco na região periapical do dente 21 , que estava tratado endodonticamente, e com o dente 22 ausente (Figura 3: A e B). 


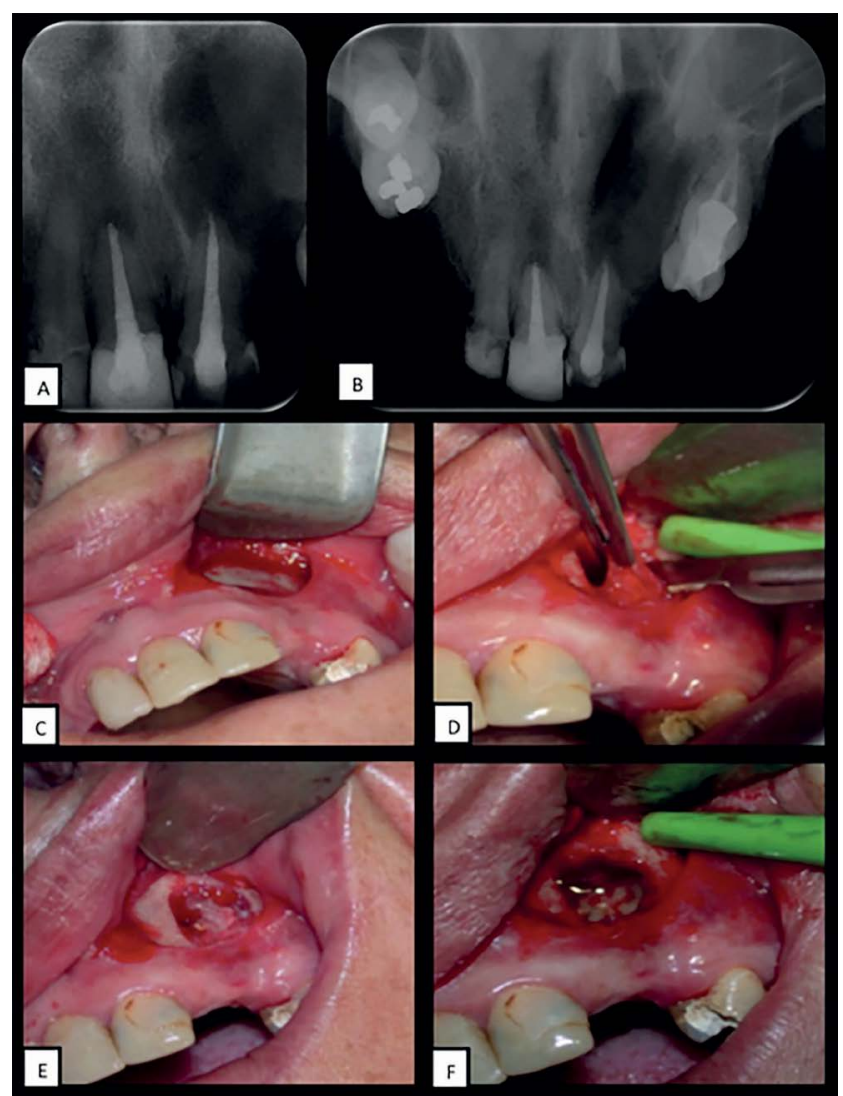

Figura 3 - Radiografia periapical (A) e oclusal (B) inicial. Acesso à lesão $(C)$, cápsula da lesão $(D)$, retirada de fragmento para exame histopatológico $(E)$, loja cirúrgica aberta $(F)$

Diante desses achados, as hipóteses diagnósticas foram de cisto periapical inflamatório oriundo do dente 21 ou cisto residual oriundo do dente 22 ausente. Considerando a extensão e a localização da lesão, optou-se por realizar a marsupialização (Figura 3: C, D, E, F).

O mesmo esquema de irrigação e higienização realizado no primeiro caso foi aplicado a essa paciente. Um fragmento da cápsula cística foi enviado para análise microscópica e revelou diagnóstico compatível com cisto de natureza inflamatória.

No pós-operatório de 45 dias, foi possível observar sinais radiográficos de neoformação óssea na região. Foi realizado então o acompanhamento radiográfico mensal e, ao final do período em que a loja cirúrgica permaneceu aberta, pôde-se observar uma significativa neoformação óssea. No controle de três anos, foi verificado o total reparo da região (Figura 4).

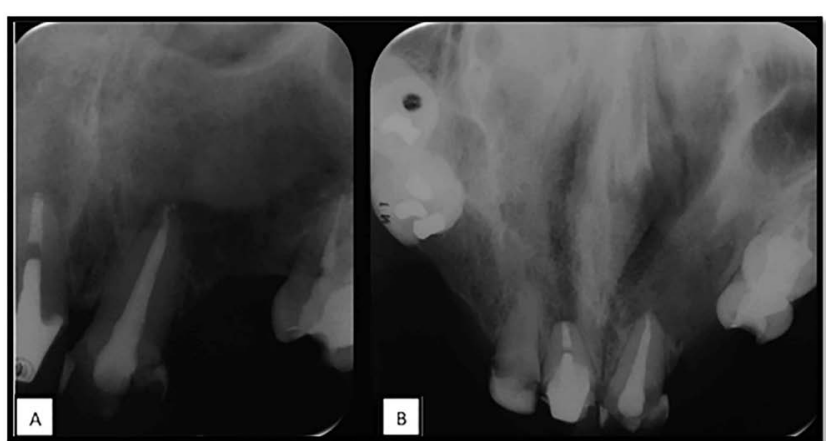

Figura 4 - Radiografia periapical (A) e oclusal (B) no pós-operatório de três anos demonstrando a evolução favorável do caso

\section{Discussão}

O tratamento do cisto periapical inflamatório é discutido há muito tempo. Alguns autores defendem que apenas o tratamento endodôntico do dente afetado seria suficiente ${ }^{5-8}$, enquanto outros acreditam na necessidade de intervenção cirúrgica ${ }^{9,10}$. No caso dos cistos dentígeros e residuais, é consenso a intervenção cirúrgica para resolução do caso.

Há autores que defendem a descompressão seguida de enucleacão. Contudo, a instalação de um artefato para manutenção da janela cirúrgica pode causar incômodo ao paciente, assim como pode converter-se em um foco retentor de placa bacteriana. Já a enucleação de lesões extensas pode comprometer dentes e estruturas anatômicas bem como causar danos a nervos e vasos da região $0^{8,10-12}$.

Outra opção de tratamento é a marsupialização - janela cirúrgica suturada junto à mucosa bucal - seguida de um segundo tempo cirúrgico de enucleação. A marsupialização é aconselhável para prevenção de possíveis complicações, pois, além de conservadora e pouco invasiva, pode manter o dente não irrompido e promover seu irrompimento ${ }^{10,13-15}$.

Alguns pesquisadores acreditam que apenas com a marsupialização possa ocorrer total resolução da lesão. Uma grande vantagem desse procedimento é a possibilidade da ausência de um segundo tempo cirúrgico, reduzindo a morbidade para o paciente. Duas formas de resolução são consideradas: o epitélio pode sofrer metaplasia escamosa para tornar-se uma mucosa normal ${ }^{1,16}$ ou ainda pode sofrer substituição, quando as bordas da lesão dão origem a um epitélio normal, substituindo subsequentemente o epitélio cístico ${ }^{1}$.

A grande maioria dos trabalhos relacionados à marsupialização como tratamento definitivo aborda o tumor odontogênico queratocístico (TOQ) - anteriormente classificado como cisto e denominado queratocisto odontogênico ${ }^{1,4,17}$. Por apresentarem comportamento agressivo e recidivante, muitas vezes, abordagens mais invasivas seguidas de tratamentos adjuvantes (como a crioterapia e aplicação da solução de Carnoy) são requeridos, e a marsupialização como único método de tratamento pode não ser efetiva. 
Uma retratação realizada por Pogrel $(2006)^{18}$ discorreu sobre a recidiva de alguns casos de TOQ em seu estudo prévio. $\mathrm{O}$ autor mostrou que nem sempre a marsupialização nos casos de TOQ é eficiente, e uma possível causa dessa recorrência é devido à presença de cistos "satélites", que permanecem mesmo após a marsupialização ${ }^{18}$. Apesar de o índice de recorrência ter sido muito pequeno, ele deve ser levado em consideração. Contudo, trabalhos em que a técnica foi aplicada para lesões de comportamento menos agressivo, como o cisto dentígero ${ }^{4,19,20}$, demostraram resultados satisfatórios.

Não foram encontrados na literatura trabalhos que abordassem as diferenças entre procedimentos de marsupialização ou descompressão em cistos odontogênicos de origem inflamatória ou de desenvolvimento. Como mencionado, a maioria dos trabalhos investiga os TOQs, tumores com altas taxas de recidiva. Por se tratar de cistos com bom prognóstico pós-tratamento cirúrgico, acredita-se que a marsupialização seja viável nesses casos, desde que o tratamento endodôntico seja realizado, no caso de cistos periapicais inflamatórios.

Entretanto, é importante enfatizar as limitações dessa técnica, como a impossibilidade de analisar microscopicamente toda a extensão da lesão, a necessidade de períodos mais longos para o tratamento e a dependência da colaboração dos pacientes quanto à higienização da cavidade, fator essencial para o sucesso da técnica ${ }^{1,4,16,20}$. Outro ponto importante a ser destacado é a necessidade de uma abertura ampla da loja cirúrgica, para que ela não se feche e o fluido cístico volte a se acumular, incorrendo na necessidade de um segundo tempo cirúrgico para nova abertura ${ }^{14,21,22}$, o que já descaracterizaria a marsupialização como um procedimento isolado.

Apesar do sucesso obtido em ambos os casos, a marsupialização isolada não deve ser considerada como um procedimento padrão. Os controles clínicos e radiográficos subsequentes são fundamentais na decisão em submeter ou não o paciente à subsequente cirurgia de enucleação. Além disso, nos casos em que a comunicação mantém-se aberta após total regressão da lesão, o fechamento cirúrgico dessa fístula é necessário, ou seja, uma segunda cirurgia é realizada de qualquer maneira. Seguramente, quando possível, a enucleação ainda é o tratamento de escolha, visto que possibilita a análise microscópica de toda a lesão, menor morbidade ao paciente e resolução mais rápida para o caso.

Nos casos clínicos apresentados, a marsupialização como tratamento definitivo mostrou-se como uma técnica favorável para os tipos de lesões abordados, uma vez que houve reparo ósseo satisfatório e ausência de sinais de recidiva, sem necessidade de um segundo procedimento cirúrgico. É importante ressaltar que a colaboração dos pacientes e os controles radiográficos periódicos foram essenciais no sucesso do tratamento.

\section{Considerações}

De acordo com a literatura consultada e considerando a evolução dos casos clínicos apresentados, conclui-se que a marsupialização pode ser utilizada como tratamento definitivo em determinados cistos odontogênicos desde que a técnica seja corretamente executada e que haja colaboração do paciente. A patogenia da lesão também parece exercer impacto no sucesso desse tipo de tratamento e o comportamento biológico da patologia em questão deve ser considerado.

\section{Agradecimentos}

Os autores agradecem ao prof. Dr. Heliton Gustavo de Lima pela valiosa contribuição neste trabalho.

\section{Abstract}

Objective: Marsupialization is a treatment option for cysts, which a surgical window communicating with the oral cavity and sutured along the adjacent mucosa is performed for the progressive emptying of the internal content of the lesion, resulting in its decompression and consequent size reduction. By definition, the technique is followed by a second surgical time for the resection of the remaining lesion. This article aims to present a literature review regarding the marsupialization technique, and report two cases of odontogenic cysts from different origins, in which marsupialization was performed as definitive treatment with satisfactory results in both cases. Case report: (1) Man, 22 years old, with a well-defined radiolucent area involving the crown of tooth 48 suggestive of a dentigerous cyst. The patient underwent extraction of tooth 48 and marsupialization, with total remission of the lesion. (2) Woman, 38 years old, referred by the endodontist due to the presence of a well-defined radiolucent lesion with radiopaque halo in the periapical region of tooth 21 suggestive of a cyst with inflammatory origin. Marsupialization was the treatment selected, resulting in bone repair and lesion remission. Final considerations: Marsupialization as a definitive treatment for cystic lesions is an approach that reduces patient morbidity, and preserves noble anatomical structures, but such topic is rarely addressed in the literature. It is worth noting that this isolated procedure is not predictable and the outcome of each case should be evaluated.

Keywords: Dentigerous cyst. Periapical cyst. Oral surgery.

\section{Referências}

1. Pogrel MA, Jordan RCK. Marsupialization as a definitive treatment for the odontogenic keratocyst. J Maxillofac Oral Surg 2004; 62(6):651-5.

2. Peterson L. Cirurgia oral e maxilofacial contemporânea. 3. ed. Rio de Janeiro: Elsevier; 2000. 
3. Hanada K, Yoshimura Y, Sugata T, Yoshiga K, Takahashi Y, Seyama A et al. Clinical investigation of marsupialization for the cystic lesion of jaw. J Maxillofac Oral Surg 1983; 41(4):274-6.

4. Bastos EG, Cruz MCFN, Martins GAS, Mendes MC, Marques RVCF. Marsupialização de cisto dentígero na mandíbula em uma criança de sete anos de idade na dentição mista: relato de caso. Rev Odontol Unesp 2011; 40(5):268-71.

5. Bhaskar SN. Nonsurgical resolution of radicular cysts. Oral Surg Oral Med Oral Pathol 1972; 34(3):458-68.

6. Shah N. Nonsurgical management of periapical lesions: a prospective study. Oral Surg Oral Med Oral Pathol Oral Radiol 1988; 66(3):365-71.

7. Valois CRA, Costa-Júnior ED. Periapical cyst repair after nonsurgical endodontic therapy - case report. Braz Dent J 2005; 16(5):254-8.

8. Vasconcelos RG, Queiroz LMG, Alvez-Júnior LC, Germano AR, Vasconcelos MG. Abordagem terapêutica em cisto radicular de grandes proporções: relato de caso. Rev Bras Ciênc Saúde 2012; 16(3):467-74

9. Neaverth EJ, Burg HA. Decompression of large periapical cystic lesions. J Endod 1982; 8(4):175-82.

10. Henry-Neto MDE, Barbosa DZ, Silva CJ, Silva MCP. Marsupialização e enucleação de cisto radicular apical. Rev Inpeo Odontol 2007; 1(1):33-8.

11. Araújo A, Gabrielli MFR, Medeiros PJ. Aspectos atuais da cirurgia e traumatologia bucomaxilofacial. São Paulo: Livraria Santos; 2007.

12. Brandt-Filho SHO, Marzola C, Toledo-Filho JL, Pereira LC, Pastori CM, Zorzetto DLG et al. Técnica da descompressão para o tratamento cirúrgico dos cistos da cavidade bucal. Rev Odontol ATO 2010; 10(5):254-66.

13. Jones TA, Perry RJ, Wake MJ. Marsupialization of a large unilateral mandibular dentigerous cyst in a 6-year-old boy a case report. Dent Update 2003; 30(10):557-61.

14. Tolentino ES, Tolentino LS, Camarini ET, Iwaki-Filho L, Iwaki LCV. Marsupialização de extenso queratocisto odontogênico em região anterior de mandíbula. Rev Bras Odontol 2008; 65(2):224-7.

15. Blaya DS, Blaya MG, Menezes JDS, Silva CO, Perez WB, Oliveira MG. Cisto dentígero mandibular tratado com marsupialização e enucleação: relato de dois casos. Rev Bras Cir Bucomaxilofac 2010; 10(2):99-104.

16. Tabrizi R, Ozkan BT, Dehgani A, Langer NJ. Marsupialization as treatment option for the odontogênico keratocyst. J Craniofac Surg 2012; 23(5):459-61.

17. Neville BW. Patologia oral \& maxilofacial. 2. ed. Rio de Janeiro: Guanabara Koogan; 2004.

18. Pogrel MA, Jordan RC. Retratação em: Pogrel MA. J Oral Maxillofac Surg 2006; 9(32):362-3.

19. Deboni MCZ, Brozoski MA, Traina AA, Acay RR, Naclério-Homem MG. Surgical management of dentigerous cyst and keratocystic odontogenic tumor in children: a conservative approach and 7-year follow-up. J Appl Oral Sci 2012; 20(2):282-5.

20. Carreira M, Dantas DB, Marchionni AM, Oliveira MG, Andrade MGS. Conservative treatment of the dentigerous cyst: report of two cases. Braz J Oral Sci 2013; 12(1):52-6.

21. Teixeira RG, Moraes PC, Jodas CRP, Bonecker MJS, Tacchelli DP. Decompression of a maxillary dentigerous cyst. Rev Gaúcha Odontol 2011; 59(2):299-303.

22. Pogrel MA. Treatment of keratocysts: The case for decompression and marsupialization. J Oral Maxillofac Surg 2005; 63(11):1667-73.

\section{Endereço para correspondência:}

Jéssica Araújo Figueira

Av. Prudente de Moraes, 772, apto. 401

87020-010 Maringá, PR, Brasil

Telefone: (44) 9125-0078 / 3025-4622

E-mail: jessica.a.figueira@gmail.com

Recebido: 14/07/15. Aceito: 22/10/15. 